\title{
Eyecciones coronales de masa solar a una unidad astronómica
}

Maribel S. Guerrero Vásquez

\section{Resumen}

La presente investigación concluye estadísticamente que las Eyecciones Coronales de Masa solar (CME por sus siglas en inglés), son grandes erupciones de campos magnéticos coronales solares y plasma (considerado el cuarto estado de la materia), que alcanzan la órbita de la Tierra, con características especiales de velocidad en este caso específico estudiado, se convierten en Tormentas geomagnéticas, sean de alta o baja velocidad de las CME. Para desarrollar la presente investigación se estudiaron los datos de eyecciones coronales de masa solar, con propiedades delimitadas; de velocidad, masa, y diferentes tipos de energía del año 1990 al 2012, que pudieron convertirse en tormentas geomagnéticas. Se enfoco la investigación en eventos rápidos con un rango de tiempo mayor o igual a 0.5 días y menor o igual a 1 día, para calcular el tiempo de llegada al planeta Tierra en días; se uso la distancia promedio entre nuestro planeta y el Sol. De los datos de CME de altas velocidades tenemos como resultados pocas fluctuaciones del campo magnético, o sea pocas tormentas geomagnéticas; pero se observaron valores de índices $\mathrm{k}$ desde 0 a 9 . En general haciendo un análisis comparativo de los resultados de la caracterización de Eyecciones coronales con la actividad solar, se observó que los ciclos de máxima actividad fueron atípicos, concluyendo que realmente los ciclos mínimos se han prolongado, se resalta la tendencia decreciente en la actividad solar y como consecuencia también hay tendencia decreciente en la ocurrencia de eyecciones coronales de masa que son la principal causa de las tormentas geomagnéticas.

Palabras claves: tormentas geomagnéticas, eyecciones coronales de masa (CME), plasma, ondas de choque, velocidad, masa, energía, Sol, actividad, satélites.

\section{Abstract}

This research concludes that statistically Coronal Mass Ejections sun (CME for its acronym in English), are large eruptions of solar coronal magnetic fields and 
plasma (considered the fourth state of matter) that reach the Earth's orbit, with special features to speed in this specific case study, become Geomagnetic Storms, whether high or low speed. To develop these research data coronal mass ejections of solar, with defined properties were studied; speed, mass, and different types of energy from 1990 to 2012, which could become geomagnetic storms. Research is focused on speed events with a range of greater than or equal to 0.5 days and less than or equal to one days time to calculate the time of arrival to Earth in days, using the average distance between Earth and the sun. From data CME speeds; results have as few fluctuations of the magnetic field, ie few geomagnetic storms; but $k$ indexes values were observed from 0 to 9 . In general making a comparative analysis of the results of the characterization of CMEs with solar activity, it was observed that the peak cycles were atypical, concluding that actually the minimum cycles they have long, downward trend in solar activity is highlighted and as a result there are decreasing trend in the occurrence of coronal mass ejections are the main cause of geomagnetic storms.

Keywords: geomagnetic storms and coronal mass ejections (CME), shock waves, speed, mass, power, sun, satelites.

Maribel S. Guerrero Vasquez, (Marsuy3000@yahoo.com), Departamento de Astronomía y Astrofísica, Facultad de Ciencias Espaciales,Universidad Nacional Autónoma de Honduras - UNAH 


\section{Introducción}

La importancia de poder predecir el clima global de la Tierra de acuerdo al clima espacial, es un objetivo implícito en toda investigación de la física solar y su relación con este. Del comportamiento del clima de la Tierra se desprende la relevancia social y económica, de mitigar posibles consecuencias globales por daños en la tecnología satelital entre otros. Con las observaciones de varios satélites, en la actualidad se registran continuamente durante 24 horas algunos de los fenómenos en la Corona solar (Figura 1a) como las eyecciones coronales de masa solar (CME) (Figura 1b) en diferentes longitudes de onda; a la vez ocasionan variabilidad en el clima espacial y tormentas geomagnéticas, que son perturbaciones temporales de la magnetosfera terrestre (Figura 1c). La radiación que penetra la atmosfera forma las auroras boreales y australes que son los indicadores visibles de la presencia real de la radiación recibida.; el efecto de la llegada al planeta Tierra de más o menos radiación del Sol, es evidente que influencia directamente la variabilidad climática, reflejada en la temperatura como indicador del dominio del Sol. Las CME son ondas de choque que llegan entre 24 y 36 horas después del suceso, esto solamente ocurre si la onda de choque viaja hacia la Tierra. La presión sobre la magnetosfera aumentará o disminuirá en función de la actividad solar; la presión modifica las corrientes eléctricas en la ionosfera. Las tormentas magnéticas duran de 24 a 48 horas, aunque pueden prolongarse varios días, los cambios en los campos geomagnéticos afectan negativamente la atmósfera superior y la baja atmósfera, destruyen células vivientes y producen cambios climáticos, las partículas muy energéticas afectan las partes electrónicas de los satélites; así mismo, las grandes tormentas geomagnéticas causan cambios en su órbita, provocando la caída y/o pérdida de éstos (M. Guerrero 2012).

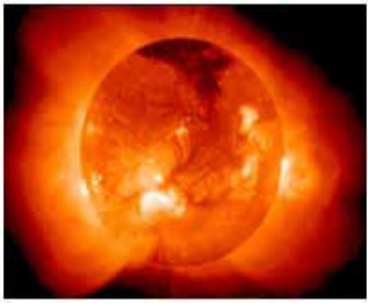

(a)

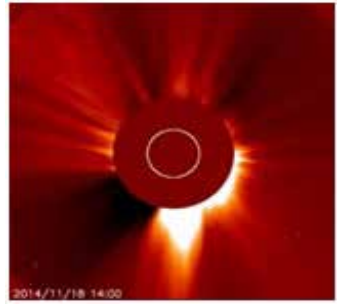

(b)

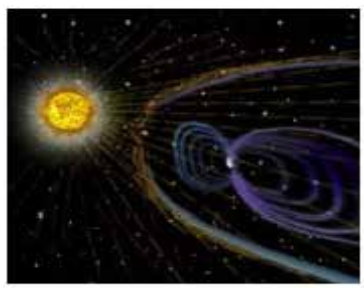

(c)

Figura 1 (a) imagen de la Corona solar en rayos X, emitiendo radiación, (b) LASCO C2 (coronografo2) muestra una eyección de masa coronal (CME)), (c) la imagen artística de la protección de la magnetosfera y la influencia del Sol (cortesía NASA-ESA). 


\section{Metodología}

De las mediciones de los fenómenos naturales en este caso fenómenos solares como las Eyecciones Coronales Solares de masa ( $\mathrm{CME}$ ), con sus variables escogidas aquí como la velocidad, aceleración, masa, diferentes tipos de energía escogidas; las Tormentas Geomagnéticas con sus indicadores los índices K, 0 sea las mediciones del campo magnético terrestre tocado entre otras necesarias para concretar la investigación cualitativamente y cumplir con el objetivo de correlacionar las CME con tormentas geomagnéticas. Se buscaron, seleccionaron y archivaron los datos digitales satelitales del satélite Solar and Heliospheric Observatory $\mathrm{SOHO}$ las $\mathrm{CME}$, del medio interplanetario que llegan a la Tierra, para transformarse en tormentas geomagnéticas. Donde la información corresponde a los años 1996 al 2012 y describe las características de los datos observados antes mencionados. Luego se hicieron los cálculos de velocidades promedio, entre las velocidades lineales y velocidades de segundo orden. Para calcular el tiempo de llegada al planeta en días de cada evento. Se utilizó la distancia promedio entre la Tierra y el Sol 149, 500,000 Km. Se construyeron tablas de CME con altas velocidades, para enfocar la investigación en eventos rápidos con un rango de tiempo mayor o igual a 0.5 días y menor o igual a 1 día. Consecuentemente se elaboraron las tablas de Tormentas geomagnéticas moderadas, fuertes, severas y extremas, correlacionadas en el tiempo de llegada a la Tierra y las eyecciones coronales solares que se produjeron, para recorrer 1 unidad astronómica. Se computaron las estadísticas, de los datos de la época seleccionada y se graficaron esos resultados. Para concluir después del análisis de los resultados estadísticos.

\section{Resultados}

Tabla 1. CME Y TG 1996- 2012

\begin{tabular}{|l|c|c|c|l|l|}
\hline ANOS & $\begin{array}{l}\text { CANTIDAD DE } \\
\text { CME } \\
\text { ENCONTRADAS }\end{array}$ & $\begin{array}{l}\text { 10SELECCION } \\
\text { CME }\end{array}$ & $\begin{array}{l}\text { 0.53 dias < T Leg } \\
\text { CMECARACTERIZADAS }<=<=1 \\
\text { DIA }\end{array}$ & \% HALOS & $\begin{array}{l}\text { TORMENTAS } \\
\text { GEOMAGETICAS } \\
\text { ENCONTRADAS }\end{array}$ \\
\hline $1996-2004$ & 9943 & 458 & 61 & 77 & 13,140 \\
\hline $2005-2012$ & 9190 & 156 & 19 & 88 & 11,680 \\
\hline TOTAL & & & 80 & $83 \%$ & 24,820 \\
\hline
\end{tabular}


En la tabla 1 observamos la cantidad de Eyecciones Coronales Solares (CME) acontecidas que se reportaron, desde el año 1996 al 2012, en la página web del satélite SOHO, $9943 \mathrm{CME}$, de las que se seleccionaron 458 con velocidad mayor a $450 \mathrm{~km} / \mathrm{s}$ que es la velocidad promedio del viento solar que llega aproximadamente en 4 días a la órbita de la Tierra. Dentro del rango de tiempo planificado discriminante en esta investigación, mayor o igual 0.53 días y menor o igual a 1 día que salieron al espacio interplanetario en un total de $80 \mathrm{CME}, 61$ entre 1996 - 2004 y 19 CME del 2005 al 2012. Aunque se esperaría que los Halos (cuando las CME sobrepasan los 1800 en el campo de visión del telescopio) fuesen proporcionales a la cantidad de eyecciones coronales, resultaron sorprendentes las cifras debido a que no hay eventos solo de Halos, con la regla discriminatoria de velocidad, explicada arriba. Los resultados muestran que hubieron mas CME rápidas entre 1996 y 2004 (61) que del 2005 al 2012 (19).

Por otra parte la cantidad de Tormentas Geomagnéticas (columna 6) arroja valores mayores que el de las CME, lo que significa es que otras CME menos rápidas, quedaron fuera de las estadísticas por no cumplir el requerimiento de tener una velocidad mayor a $450 \mathrm{~km} / \mathrm{s}$; que llegaron en forma más lenta al planeta pero que finalmente tocaron su campo magnético.

Tabla 2. COINCIDENCIAS CME Y TG moderadas, fuertes, severas y extremas 1996- 2012

\begin{tabular}{|l|l|l|l|l|l|}
\hline Años & $\begin{array}{l}\text { Cantidad de } \\
\text { CME } \\
\text { encontradas }\end{array}$ & \multicolumn{4}{l|}{$\begin{array}{l}\text { Resumen de Coincidencias } \\
\text { CME (20 Selección ) -Tormentas Geomagneticas (Indices k) }\end{array}$} \\
\hline & & $\begin{array}{l}\text { K=6 } \\
\text { G2 } \\
\text { Tormentas } \\
\text { moderadas }\end{array}$ & $\begin{array}{l}\text { K=7 } \\
\text { G3 } \\
\text { Tormenta } \\
\text { s fuertes }\end{array}$ & $\begin{array}{l}\text { K=8 } \\
\text { G4 } \\
\text { Tormentas } \\
\text { severas }\end{array}$ & $\begin{array}{l}\text { K=9 } \\
\text { G5 } \\
\text { Tormentas } \\
\text { Extremas }\end{array}$ \\
\hline \begin{tabular}{l|l|l|l|l|}
$1996-$ \\
2004
\end{tabular} & 9943 & 24 & 9 & 9 & 3 \\
\hline $\begin{array}{l}2005- \\
2012\end{array}$ & 9190 & 13 & 10 & 4 & 3 \\
\hline TOTAL & & 37 & 19 & 13 & 6 \\
\hline
\end{tabular}

En la Tabla 2, se muestran las Tormentas geomagnéticas más peligrosas expresadas por los índices $K$ con los valores más altos establecidos de 6 a 9, cabe recordar que los índices utilizados son los valores reportados para altas latitudes. Observamos que los acontecimientos van en orden decreciente, se presentaron en total 13 severas, 19 extremas y 37 moderadas, solo 6 tormentas muy peligrosas extremas con índice $k=9$ y correspondiente a $500 \mathrm{nT}$ (nano teslas). Acontecieron 
tanto en las CMEs lentas y muy rápidas, estas tormentas peligrosas, dentro de la baja actividad solar de los últimos años.

Tabla 3. Caracterización de cme a tormentas geomagnéticas

\begin{tabular}{|c|c|c|c|c|c|c|c|c|c|c|c|}
\hline $\begin{array}{l}\text { Caracteri } \\
\text { zación }\end{array}$ & $\begin{array}{l}\text { Angul } \\
\text { o de } \\
\text { posici } \\
\text { ón } \\
\text { central }\end{array}$ & $\begin{array}{l}\text { Ampli } \\
\text { tud } \\
\text { Angul } \\
\text { ar } \\
\text { (grad } \\
\text { os) }\end{array}$ & $\begin{array}{l}\text { Velo } \\
\text { ci } \\
\text { dad } \\
\text { Line } \\
\text { al } \\
\mathrm{km} / \mathrm{s}\end{array}$ & $\begin{array}{l}\text { Vel } \\
\text { oci } \\
\text { dad } \\
\text { de } \\
\text { seg } \\
\text { und } \\
\text { o } \\
\text { ord } \\
\text { en. } \\
\text { fina } \\
\text { I } \\
{[\mathrm{km}} \\
\text { ls] }\end{array}$ & $\begin{array}{l}\text { Vel } \\
\text { oci } \\
\text { dad } \\
\text { Pro } \\
\text { me } \\
\text { dio } \\
{[\mathrm{km} /} \\
\text { s] }\end{array}$ & $\begin{array}{l}\text { Tiem } \\
\text { po de } \\
\text { llega } \\
\text { da a } \\
\text { la } \\
\text { Tierra } \\
\text { en } \\
\text { días }\end{array}$ & $\begin{array}{l}\text { Tiem } \\
\text { po de } \\
\text { Ilega } \\
\text { da a } \\
\text { la } \\
\text { Tierr } \\
\text { a } \\
\text { en } \\
\text { horas }\end{array}$ & $\begin{array}{l}\text { Velo } \\
\text { cida } \\
\text { d a } \\
20 \\
\text { Radi } \\
\text { os } \\
\text { sola } \\
\text { res } \\
\text { [km/ } \\
\text { s] }\end{array}$ & $\begin{array}{l}\text { Acele } \\
\text { ra } \\
\text { ción } \\
, \mathrm{Km} / \mathrm{s} \\
{ }_{2}\end{array}$ & $\begin{array}{l}\text { Masa } \\
\text { [gram } \\
\text { os] }\end{array}$ & $\begin{array}{l}\text { Ener } \\
\text { gia } \\
\text { Cinet } \\
\text { ica } \\
\text { (ergi } \\
\text { os) }\end{array}$ \\
\hline $\begin{array}{l}\text { Valores } \\
\text { Máximos }\end{array}$ & $\begin{array}{l}\text { Halo } \\
\text { (OA) }\end{array}$ & 360 & $\underline{3387}$ & $\frac{328}{4}$ & $\begin{array}{l}3264 \\
.5\end{array}$ & $\begin{array}{l}0.530 \\
0\end{array}$ & 16.30 & 1124 & $\begin{array}{l}198.0 \\
1\end{array}$ & $\begin{array}{l}1.9 \times 1 \\
0^{32}\end{array}$ & 359 \\
\hline $\begin{array}{l}\text { Valores } \\
\text { Mínimos }\end{array}$ & 2 & 24 & 218 & 280 & 278 & 1.005 & 24.12 & $\begin{array}{l}- \\
179 . \\
7\end{array}$ & -159.1 & $\begin{array}{l}2.70 x \\
10^{29}\end{array}$ & 15 \\
\hline
\end{tabular}

En la tabla 3 observamos las características principales para que las eyecciones coronales solares (CME), que puedan llegar a tocar el campo magnético de la Tierra o sea convertirse en Tormentas Geomagnéticas (TG). En forma resumida observamos que el valor máximo para ángulos de posición central es un Halo OA (con esquema de asimetría), por lo tanto su amplitud angular es igual a 360 grados, Cada CME se caracteriza por tres velocidades: 1. la velocidad lineal (o ajuste polinomial) obtenida ajustando una línea recta, para las mediciones en tiempo 2. La velocidad cuadrática obtenida mediante el ajuste de una parábola (cuadrática o de segundo orden ajuste polinómial ) para las mediciones en tiempo altura y la evaluación de la velocidad en el momento final ( última posible medida de la altura) y 3. La velocidad obtenida como en (2) pero evaluada cuando la CME está a una distancia de 20 radios solares. Desde el momento de la medición de la altura final varía de un evento a otro, la velocidad de 20 radios solares es útil para comparar diferentes velocidades. Se debe tener precaución con el método de las CMEs que se desvanecen antes de llegar a 20 radios solares. Su velocidad lineal $3387 \mathrm{~km} / \mathrm{s}$, es la más alta encontrada en el rango de tiempo estudiado 1996-2012, la velocidad de segundo orden es muy alta igual a $3284 \mathrm{~km} / \mathrm{s}$. El promedio de ambas resultó igual a $3264.5 \mathrm{~km} / \mathrm{s}$. 
Conociendo la velocidad promedio y la distancia promedio se calculó el tiempo promedio que tardó en llegar esa eyección de masa coronal a la Tierra, el resultado es de 0.53 días o sea 16.3 horas. Otra característica es la velocidad de la CME a 20 radios solares de distancia más allá de la órbita de Urano, antes de Neptuno aún sigue siendo un evento rápido La aceleración de una CME puede ser positiva, negativa o cercana a cero significando que una CME se acelera, se mueva con velocidad constante o disminuya la velocidad en la observación del telescopio de LASCO. Se necesita un mínimo de tres mediciones en tiempo de altura para una estimación de la aceleración, pero la precisión aumenta cuando hay más mediciones ( Howard, R. A et Al, 1985). Las aceleraciones con sólo tres mediciones no son confiables por lo tanto no se reportan datos. . Observamos en la tabla 3 columna 10 una aceleración alta, positiva igual a $1980 \mathrm{~km} / \mathrm{s} 2$. Cada CME también se caracteriza por una masa y una energía cinética. En general, existen grandes incertidumbres en estos números. La estimación de la masa de una CME implica una serie de supuestos, por lo que los valores dados deben ser tomado como representativos (Miklenic et al.2011). Por ejemplo, la mayoría de las CME muestran un aumento de la masa cuando atraviesan los primeros radios solares y a continuación, la masa alcanza un valor casi constante. Este valor constante se toma como la masarepresentativa para este caso vemos en la columna $11 \mathrm{el}$ dato de la masa para el evento más rápido igual a 1.9×10+32 gramos y la energía cinética igual a 359 ergios. En esta fecha 2004/11/10 se reportaron tormentas con índices $\mathrm{k}$ de 6,7 y 8 ninguna con $\mathrm{k}$ igual a 9 .

Para el evento más lento (velocidad promedio $278 \mathrm{~km} / \mathrm{s}$ ) dentro del rango de tiempo definido como máximo (1 día) para llegar a nuestro planeta, se encontraron otras características que muestra la tabla 3 en la fila dos, vemos que el Angulo de posición central es de 2 grados y la amplitud angular es apenas de 24 grados, la masa es grande y la energía cinética muy baja. Pero que finalmente llegó a la Tierra y se pudo convertir en Tormenta geomagnética. Abajo la figura 1 muestra la CME más rápida ocurrida el 2004/11/10, del coronógrafo C2 de LASCO a bordo del satélite $\mathrm{SOHO}$ sus respectivas graficas de velocidad, ángulo de posición y la aceleración negativa o sea que hubo desaceleración $(-159.1 \mathrm{~km} / \mathrm{s} 2)$ posiblemente debido a su gran masa entre otras variables. Penosamente en los meses de abril, mayo, junio, julio, agosto y septiembre no hubo ningún tipo de registro del NOAA. 


\section{$2004 / 11 / 10 \quad$ CME VMAX $=3,387 \mathrm{~km} / \mathrm{s}$}

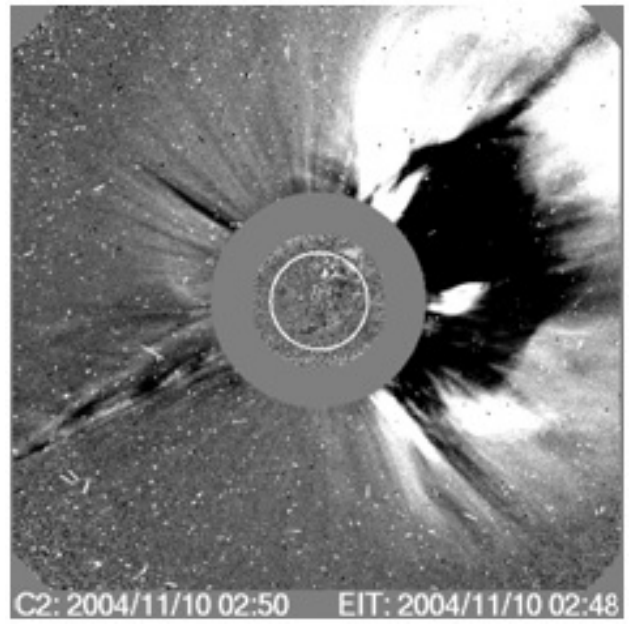

Figura 2: Imagen de una CME a las 02:48 UT, con la velocidad máxima encontrada en los datos desde 1996 al 2012 del coronógrafo C2. Fuente: LASCO / SOHO, en infrarrojo extremo.
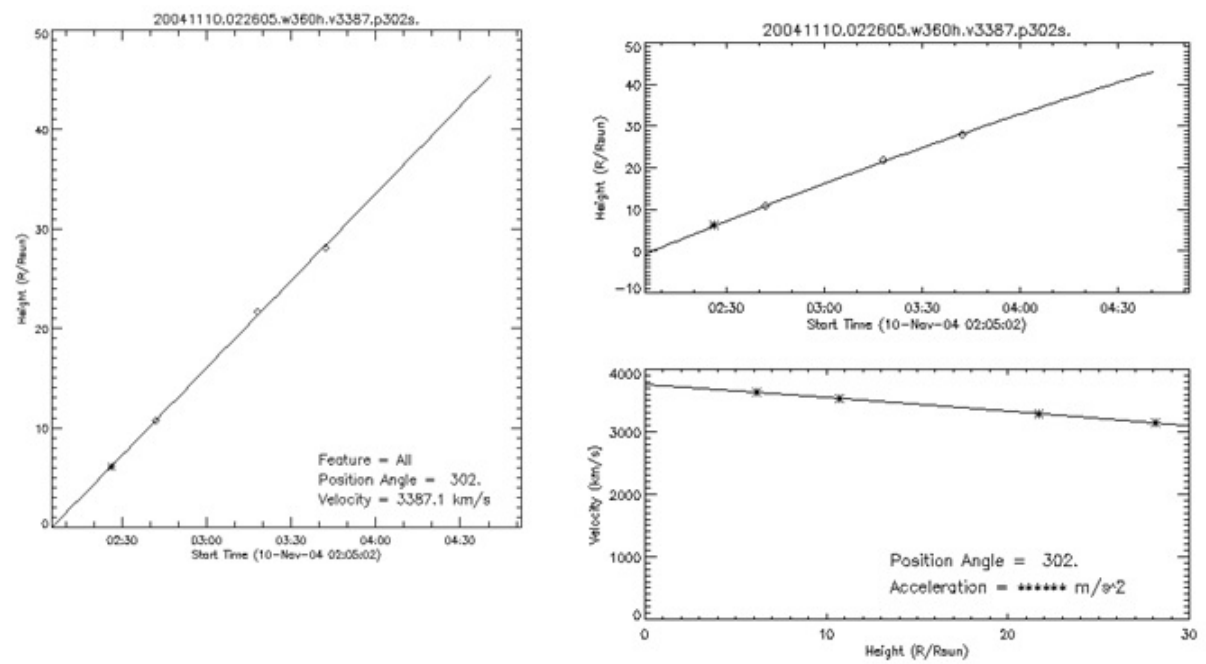

Figura 3 la grafica izquierda muestra la velocidad lineal y ángulo de posición, superior derecha el tiempo de inicio, inferior derecha ángulo de posición y la aceleración del mismo evento Fuente: http://hirweb.nict.go.jp/dimages/magneka/20041208.html 


\section{2 / 07 / $06 \quad$ Vlineal MIN=218km/}

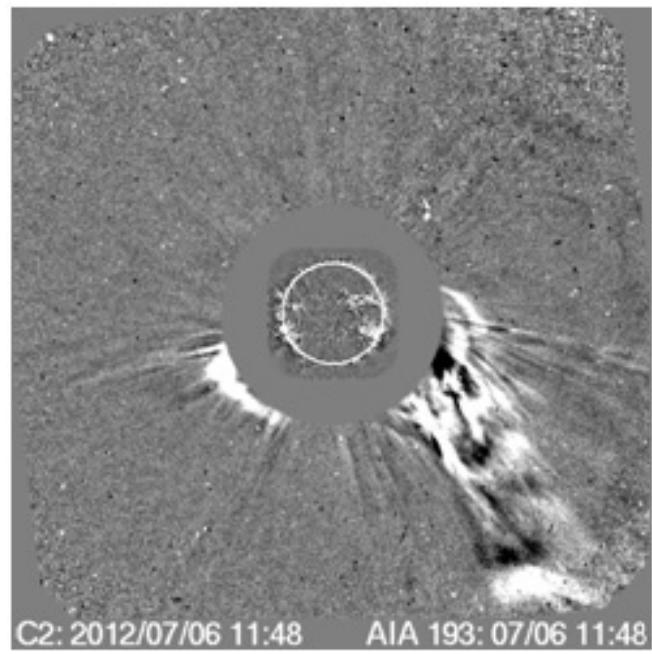

Figura 4. La Imagen del C2 muestra la CME a las 11:48. Fuente: C2 de LASCO/ SOHO i UT
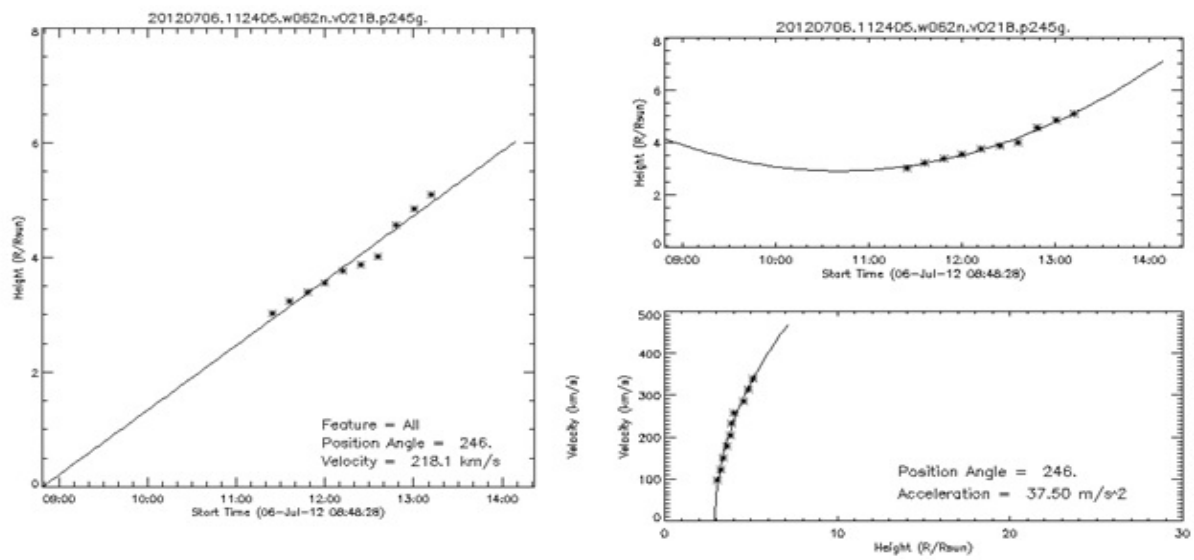

Figura 5: Gráficas: izquierda la imagen muestra la Figura VlinealMIN=218km/s, superior derecha velocidad de segundo orden, la superior derecha representa el tiempo de inicio de la CME, la inferior derecha representa la velocidad de segundo orden a 20 radios solares Fuente: http://hirweb.

nict.go.jp/dimages/magneka/20121231t 


\section{Discusión}

Un análisis comparativo muestra los resultados de la caracterización de Eyecciones coronales de masa solar con la actividad solar, donde los ciclos de máxima actividad fueron atípicos, se presentó mayor actividad en los ciclos mínimos y viceversa, concluyendo que los ciclos mínimos (Fig. 6 y 7) se han prolongado y realmente no ha habido máxima actividad, que se ha llamado máximos a los dos últimos por definición: por la posición cercana de las manchas respecto al ecuador del sol como consecuencia tenemos pocas fluctuaciones del campo magnético, 0 sea pocas tormentas geomagnéticas y escasos eventos solares en general, durante los periodos llamados de máxima actividad solar. Pero se observaron valores de índices $\mathrm{k}$ desde 0 a 9 . En forma específica para la CME máxima, vista por primera vez a las 2:26 horas, le sumamos el tiempo aproximado de 0.53 días, que tardó en llegar a la Tierra, entonces se correlaciona con la Tormenta geomagnética con índice $k=7$, ocurrida entre las 12-15 horas, reportada. De la CME más lenta estudiada no se puede concluir nada por no haber datos del satélite NOAA en esa fecha. El estudio de la caracterización por otra parte también se vio afectado por los escases de eventos muy rápidos y también lentos, en la época de investigación definida. Con los elementos estudiados se puede decir que toda eyección de masa coronal rápida llega a la Tierra produce disturbios del campo magnético, del orden de índice $\mathrm{k}$ igual o mayor a 7 .

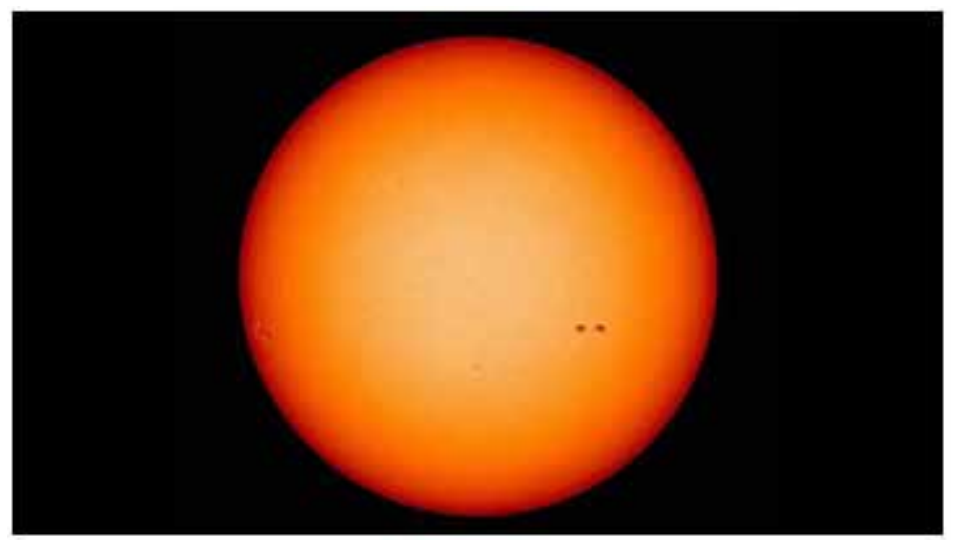

Figura 6: La imagen muestra la baja actividad del Sol, desde el 2011 existe un número de manchas solares muy por debajo de los valores previstos y las fuertes erupciones solares que se esperaban han sido muy poco frecuentes, asegura el observatorio de la NASA. 

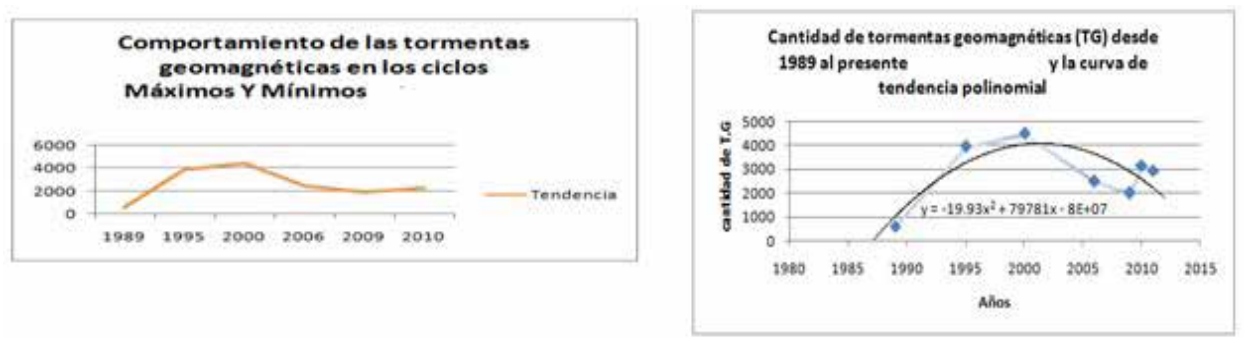

Figura 7. Izquierda la curva muestra la tendencia de tormentas geomagnéticas desde 1989 al presente, a la derecha la curva representa la tendencia lineal y polinomial descendente debido a la baja actividad del Sol. Fuente: Tesis "EL MITO DEL CALENTAMIENTO GLOBAL" M. Guerrero 2012

Tabla 4. RESUMEN COINCIDENCIAS CME -TORMENTAS GEOMAGNÉTICAS (INDICE k)

\begin{tabular}{|l|l|l|l|l|l||}
\hline & $\begin{array}{l}\text { CANTIDAD DE } \\
\text { CME } \\
\text { ENCONTRADAS }\end{array}$ & \multicolumn{4}{l|}{$\begin{array}{l}\text { RESUMEN COINCIDENCIAS } \\
\text { CME -TORMENTAS GEOMAGNÉTICAS (INDICE k) }\end{array}$} \\
\hline AŇOS & & $\begin{array}{l}\text { K=6 } \\
\text { G2 } \\
\text { Tormentas } \\
\text { moderadas }\end{array}$ & $\begin{array}{l}\text { K=7 } \\
\text { G3 } \\
\text { Tormentas } \\
\text { fuertes }\end{array}$ & $\begin{array}{l}\text { K=8 } \\
\text { G4 } \\
\text { Tormentas severas }\end{array}$ & $\begin{array}{l}\text { K=9 } \\
\text { G5 } \\
\text { Tormentas Extremas }\end{array}$ \\
\hline $1996-2004$ & 9943 & 24 & 9 & 9 & 3 \\
\hline $2005-2012$ & 9190 & 13 & 10 & 4 & 3 \\
\hline \hline TOTAL & & 37 & 19 & 13 & 6 \\
\hline
\end{tabular}

\section{Conclusiones}

En conjunto, esta investigación tiene por objeto proporcionar un análisis cuantitativo completo especifico, con la descripción de todos los aspectos principales de un evento máximo único y un evento mínimo único que comenzaron con una CME y que se esperaba que tocara la magnetosfera, consecuentemente convirtiéndose en una Tormenta Geomagnética con un valor cualquiera de la medición del índice $\mathrm{K}$ en altas latitudes, pero que pudieran tener mayor posibilidad, lo valores más altos para k iguales a 7, 8 y 9 por sus características arriba descritas. De allí se puede inferir la generalización: que todas las Eyecciones Coronales de Masa que fueron seleccionadas por ser muy rápidas, en principio; y con masas relativamente bajas terminaron convirtiéndose en Tormentas Geomagnéticas con valores de índices $\mathrm{k}$ desde 0 hasta 9 , desde tormentas menores hasta extremas. Concluyendo que no dependen de la velocidad para ser tormentas peligrosas o no pero que el clima del Sol afecta directamente el clima de la Tierra y otros planetas, es quien lo 
controla, subiendo o bajando su temperatura de acuerdo a la actividad máxima o mínima de la estrella más cercana.

\section{Bibliografía}

- Chen J. (1997). Coronal mass ejections, causes and consequences. En Geophysical Monograph. Florida United States of América, Library of congress cataloging - in publication Data Editorial

- Current Quarter Daily Geomagnetic Data, disponible noaa.gov/ftpdir/indices/old_indices/2011Q1_DSD.txt

en:http://www.swpc.

- http://www.swpc.noaa.gov/ftpdir/indices/old_indices/READMEPost-eruption Arcades PEAs have been commonly observed after nearly all CMEeruptions (Schwenn et al. 2006)

- The Astrophysical Journal, 751:21 (16pp), 2012 May 20 Landi et al.(2004) report that a one-to-one relationship existed between PEAs and CMEs in 236 events observed between 1997-2002

- PEAs are a normal expectation from theory (McKenzie \&Hudson 1999 the reconnection site that isforming new hot loops at larger heights (Lin et al. 2004). coronal manifestations of transient coronal holes appearing on the disk after CME events (Miklenic et al. 2011).

- Not all CMEs have been reported to be followed by dimmings (Reinard \& Biesecker 2008,2009), CMEs with dimmings were found to be on average faster than those without ones (Reinard \& Biesecker 2009).

- The time of their appearance has been shown to be tied to the time of CME acceleration and energy release in a flare (Miklenic et al.2011)

- The disappearance of transient coronal holes on the disk was found to be due to the shrinking of the hole region rather than the heating of local plasma (Kahler \& Hudson 2001), and Attrill et al. (2008)

- Gosling, J.T et Al, 1974, Solar Wind three; proceedings of the Third Conference, Pacific Grove, Los Angeles, University of California 
- Gosling, J.T., 1999, Journal of geophysical research, Volume 104, p. 1251512524

- Howard, R. A et Al, 1985, Journal of geophysical research, Vol 90, Recuperado de: http://www.docstoc.com/docs/58393503/An-Historical-Perspective-to-CME.

- Hundhausen, A. J, 1996, Coronal mass ejections, in cosmic winds and the heliosphere, eds. J.R. Jokipiiet al., Arizona, Tucson

- Sitio web, NOAA Space Weather Scale for Geomagnetic Storms, disponible en http://www.swpc.noaa.gov/ftpdir/lists/geomag/AK.txt http://www.swpc.noaa. gov/ftpmenu/indices/old_indices.html

- Sitio Web 2010 Daily Geomagnetic Data disponible en: http://hirweb.nict.go.jp/ dimages/magneka/19890904t

- Sitio Web, http://science.nasa.gov/heliophysics/focus-areas/magnetosphere-ionosphere/

- Sitio Web, Geomagnetic Field Data Plot, disponible en: http://kogma.nict.go.jp/ cgi-bin/geomaginterface

- Sitio Web, Geomagnetic Activity Chart, disponible en http://hirweb.nict.go.jp/ sedoss/geoact

- Sitio Web, MAGNE KA and Geomagnetic Data Plot, disponible en

- http://hirweb.nict.go.jp/dimages/magneka/19890904t

- http://crlhir.nict.go.jp/dimages/magneka/19940220.htm

- $\quad$ http://hirweb.nict.go.jp/dimages/magneka/19950101t

- $\quad$ http://hirweb.nict.go.jp/dimages/magneka/19950801t

- http://hirweb.nict.go.jp/dimages/magneka/19951111t

- http://hirweb.nict.go.jp/dimages/magneka/20000101t

- http://hirweb.nict.go.jp/dimages/magneka/20041208.html

- http://hirweb.nict.go.jp/dimages/magneka/20060301t 
- http://hirweb.nict.go.jp/dimages/magneka/20061231t

- http://www.swpc.noaa.gov/ftpmenu/indices/old_indices.html 